\title{
Adaptação e Evidências de Validade da Escala de Parâmetros da Carreira Caleidoscópica ${ }^{1}$
}

\section{Resumo}

\author{
Patrícia Bock Bandeira ${ }^{2}$ \\ Pontifícia Universidade Católica do Rio Grande do Sul, Porto Alegre, RS, Brasil \\ Alexsandro Luiz De Andrade \\ Universidade Federal do Espírito Santo, Vitória, ES, Brasil \\ Marcelo de Campos Velho Nora \\ Marianna Marques Braga \\ Manoela Ziebell de Oliveira \\ Pontifícia Universidade Católica do Rio Grande do Sul, Porto Alegre, RS, Brasil
}

O Modelo de Carreira Caleidoscópica descreve como os indivíduos alteram o desenho de suas carreiras e tomam decisões profissionais segundo os parâmetros de Autenticidade, Balanço e Crescimento. O objetivo deste trabalho foi adaptar e apresentar evidências de validade da Escala de Parâmetros da Carreira Caleidoscópica em uma amostra de 272 profissionais brasileiros, utilizando o procedimento de análise fatorial confirmatória, com método de estimação da máxima verossimilhança. A versão final do instrumento apresentou 19 itens, distribuídos nos fatores previstos, com os indicadores de confiabilidade ômega: Autenticidade $(\omega=, 66)$, Balanço $(\omega=, 78)$ e Crescimento $(\omega=, 79)$. Comparou-se os resultados segundo as características sociodemográficas dos participantes, reforçando a possibilidade de aplicação da teoria para o aconselhamento de carreira.

Palavras-chave: carreira; desenvolvimento profissional; avaliação psicológica; aspirações profissionais

Abstract: Adaptation and Validity Evidences of the Kaleidoscope Career Parameters Scale

The Kaleidoscopic Career Model describes how individuals alter the design of their careers and make professional decisions according to the parameters of Authenticity, Balance and Challenge. This study aimed to adapt and present validity evidence of the Kaleidoscopic Career Parameter Scale in a sample of 272 Brazilian professionals, using the procedure of confirmatory factorial analysis, with a maximum likelihood estimation method. The final version of the instrument presented 19 items, distributed in the predicted factors, with the omega reliability indicators: Authenticity $(\omega=, 66)$, Balance $(\omega=, 78)$ and Growth $(\omega=, 79)$. Finally, the results were compared according to the sociodemographic characteristics of the participants, reinforcing the possibility of applying the theory for career counseling. Keywords: career; professional development; psychological evaluation; professional aspirations

Resumen: Adaptación y evidencias de validez de la Escala de Parámetros de la Carrera Caleidoscópica

El Modelo de Carrera Caleidoscópica describe cómo los individuos alteran el diseño de sus carreras y toman sus decisiones profesionales según los parámetros de Autenticidad, Equilibrio y Crecimiento. El objetivo del trabajo fue adaptar y presentar evidencias de validez de la Escala de Parámetros de la Carrera Caleidoscópica en una muestra de 272 profesionales brasileños, utilizando el análisis factorial confirmatorio, con método de estimación de máxima verosimilitud. La versión final presentó 19 ítems, distribuidos en los factores previstos, con los indicadores de confiabilidad omega: Autenticidad $(\omega=, 66)$, Equilibrio $(\omega=, 78)$ y Crecimiento $(\omega=, 79)$. Se compararon los resultados según las características sociodemográficas de los participantes, reforzando la posibilidad de aplicación de la teoría para la asesoría de carrera.

Palabras clave: carrera; desarrollo profesional; evaluación psicológica; aspiraciones profesionales.

\footnotetext{
1 Agradecimentos: À professora phd. Sherry Sullivan, autora do instrumento original, pelo incentivo e apoio na realização deste trabalho e também à Fundação Coordenação de Aperfeiçoamento de Pessoal de Nível Superior (Ministério da Educação) pela concessão de bolsa de mestrado.

2 Endereço para correspondência: Grupo de Estudos sobre Desenvolvimento de Carreira - GEDC Programa de Pós-Graduação em Psicologia - Pontifícia Universidade Católica do Rio Grande do Sul Av. Ipiranga, 6681 - Prédio 11 - Sala 938. 90619-900 Porto Alegre - RS - Brazil - Fone/Fax +55 51 3320-7747
} 
A carreira pode ser definida como um processo que ocorre ao longo da vida e refere-se - mas não se restringe - ao desempenho de atividades profissionais, envolvendo aspectos tanto objetivos quanto subjetivos (Ambiel, 2014; Baruch \& Vardi, 2015; Rosa, Zampier, \& Stefano, 2017). Até meados do século XX, as carreiras eram tradicionalmente definidas como lineares, e se caracterizavam pelo avanço vertical na hierarquia de uma única organização ao longo da trajetória profissional dos indivíduos (Baruch, Szucs \& Gunz, 2015; Clarke, 2015; Duarte et al., 2010). No entanto, a partir da década de 1970, a dinâmica das relações de trabalho sofreu alterações significativas, decorrentes da aceleração dos avanços tecnológicos, do ingresso intenso de mulheres no mercado de trabalho, do aumento da competitividade entre empresas e das políticas governamentais de austeridade (Andrade, Oliveira, \& Hatfield, 2017; Baruch, Szucs, \& Gunz, 2015; Duarte et al., 2010; Oliveira, Zanon, Silva da Silva, Pinhatti, Gomes, \& Gauer, 2010). Diante de um cenário organizacional cada vez mais volátil e mutável, as expectativas da estabilidade no emprego e de trajetórias profissionais construídas durante a vida toda em uma só organização não puderam mais ser mantidas (Audibert \& Teixeira, 2015; Oltramari, Friederichs \& Grzybovski, 2014). Esse contexto levou empresas a adotarem estratégias de restruturação, layoffs, downsizing e terceirização, questionando a soberania dos modelos de carreira vigentes (Baruch \& Bozionelos, 2010).

A carreira tradicional tem cedido espaço a novas formas de interação entre empregados e empregadores, visto que o contrato de trabalho atual já não mais atende plenamente à expectativa de um emprego para a vida toda, ou de um desenvolvimento profissional linear e seguro. Nesse sentido, os indivíduos são incentivados a alcançar empregabilidade e progresso profissional, de forma autônoma e independentemente de uma associação duradoura com a empresa (Wilkoszynski \& Vieira, 2012). Logo, é esperado que o profissional - e não mais a organização assuma cada vez mais responsabilidade pelas decisões de carreira e busca por sucesso (DeLuca, Rocha-de-Oliveira, \& Chiesa, 2016), levando em consideração aspectos que constituem seu projeto de vida, tais como família, lazer, estudo e redes de relacionamento (Ambiel, 2014).

Embora a carreira tradicional não tenha desaparecido por completo e ainda descreva a realidade de muitos indivíduos e organizações, nas quais as movimentações são baseadas no progresso linear e hierárquico (Andrade, Kilimnik, \& Pardini, 2011; Clarke, 2013; Cordeiro \& Albuquerque, 2016), diversos modelos teóricos emergiram buscando explicar este novo contexto laboral (Baruch \& Vardi, 2015; Baruch, Szucs, \& Gunz, 2015). Ainda que haja uma diversidade de conceitos e construtos na área de carreira, pode-se destacar os modelos da carreira proteana, sem-fronteiras, resiliente, multidirecional, pós-corporativa, inteligente e caleidoscópica, pois são considerados as principais metáforas utilizadas na tentativa de sintetizar e explicar o comportamento das carreiras na contemporaneidade (Baruch \& Vardi, 2015; Baruch, Szucs \& Gunz, 2015). Dentre estes modelos explicativos, o único que leva em consideração o impacto do contexto, crenças, oportunidades, valores e relacionamentos sobre as decisões de carreira dos indivíduos ao longo da vida é o Modelo de Carreira Caleidoscópica (Sullivan \& Baruch, 2009).

Este construto, desenvolvido por Mainiero e Sullivan (2005), descreve como os indivíduos alteram o desenho de suas carreiras de acordo com os diferentes aspectos de sua vida. Assim como um caleidoscópio, que produz imagens diferentes quando seu tubo é rotacionado, indivíduos alteram o "mosaico" de suas vidas ao adequar fatores que lhes permitam uma melhor adaptação a seus papeis, necessidades e relacionamentos (Mainiero \& Sullivan, 2005; Sullivan \& Mainiero, 2007; Sullivan, Forret, Mainiero, \& Terjesen, 2007). Ou seja, profissionais avaliam as escolhas e opções de carreira disponíveis com o objetivo de determinar o melhor arranjo entre as demandas, barreiras e oportunidades de trabalho e também em relação a seus valores pessoais, interesses e relacionamentos. Ao tomar uma decisão, esta afetará a imagem formada pelo "caleidoscópio da carreira" (Mainiero \& Sullivan, 2005; Sullivan et al., 2007).

As carreiras, assim como o caleidoscópio, são dinâmicas e estão em constante mudança (Sullivan \& Mainiero, 2007). As pessoas alteram suas profissões e ocupações para ajustar-se às mudanças da vida, repensando suas prioridades profissionais para que se adequem às suas necessidades. Da mesma forma, quando uma das partes de um caleidoscópio se move, as outras também se modificam. Sendo assim, o contexto molda as escolhas, fazendo com que os indivíduos modifiquem seus "mosaicos" ao longo da vida (Sullivan \& Mainiero, 2007).

Em analogia ao caleidoscópio, que tem três espelhos, o Modelo de Carreira Caleidoscópica prevê que são três os parâmetros para a tomada de decisões de carreira: Autenticidade, Balanço e Crescimento, constituindo assim o "ABC" do Modelo de Carreira Caleidoscópica (Maniero \& Sullivan, 2005; Sullivan \& Baruch, 2009; Sullivan et al., 2007). O parâmetro Autenticidade, está associado a ser fiel a si mesmo e a seus valores, refletindo em uma maior tendência a procurar uma ocupação laboral que esteja em sintonia com os princípios de cada indivíduo, bem como o desejo de tomar decisões que abranjam da melhor forma as próprias características e valores, definindo caminhos de carreira da forma mais autêntica e significativa possível (Cabrera, 2007; Mainiero \& Sullivan, 2005). O parâmetro Balanço está relacionado ao equilíbrio entre a vida pessoal e profissional, e também à necessidade de, frente a uma decisão de carreira, analisar os possíveis impactos e consequências para o contexto e os relacionamentos pessoais, como a família por exemplo 
(Mainiero \& Sullivan, 2005). Por fim, o parâmetro Crescimento corresponde à necessidade de evolução contínua e superação de desafios profissionais, além da busca por um trabalho estimulante com foco no avanço da carreira (August, 2011; Sullivan \& Mainiero, 2008).

Cada mudança em relação aos parâmetros (Autenticidade, Balanço e Crescimento) corresponde à alteração no estilo de vida dos indivíduos e na forma como os diferentes aspectos compõem o seu contexto atual. Utilizando uma metáfora, pode-se pensar que as cores do caleidoscópio são refletidas nestes três parâmetros. Ao longo do ciclo de vida, as cores do caleidoscópio se alternam para adequarem-se ao contexto atual, fazendo com que uma cor (parâmetro) se torne mais proeminente e sirva de orientação para a tomada de decisões de carreira. Como consequência, as demais cores vão para o fundo do mosaico, mas continuam presentes, formando a imagem no caleidoscópio (Maniero \& Sullivan, 2005). Embora os demais parâmetros tenham sua influência reduzida, assumindo um papel secundário no processo decisório, eles ainda permanecem presentes e ativos, pois são relevantes para a criação do "mosaico" de vida ou de carreira de um indivíduo (Sullivan \& Baruch, 2009). Essa metáfora é demonstrada na Figura 1.

O Modelo da Carreira Caleidoscópica, portanto, reflete uma forma inovadora de se pensar a carreira contemporânea, pois leva em consideração a importância dos aspectos contextuais e pessoais, e não apenas os laborais,

\section{Imagem/Mosaico}

do Caleidoscópio
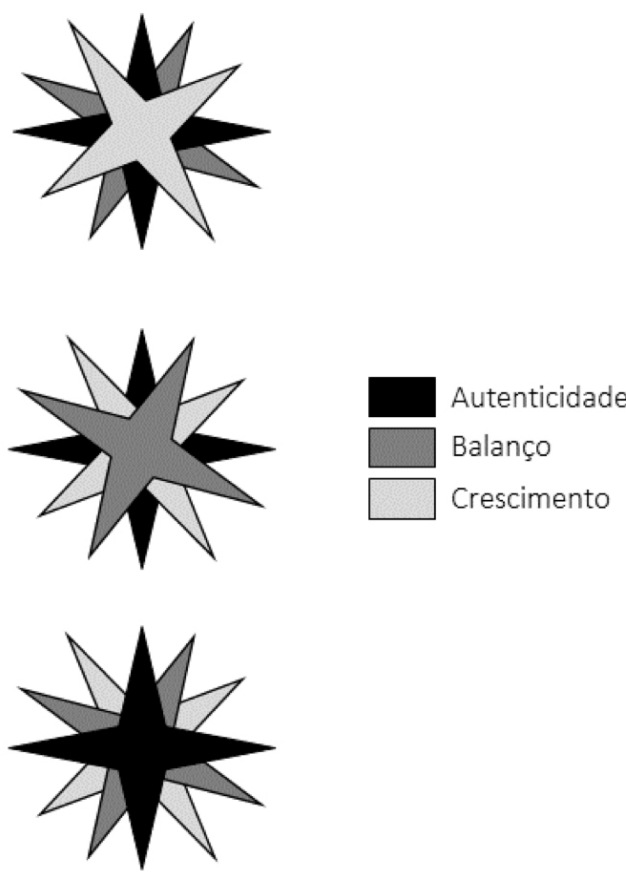

Figura 1. O Modelo de Carreira Caleidoscópica para a tomada de decisões profissionais. Ainda que hajam teorias em carreira que levem em consideração os papeis de vida desempenhados pelos indivíduos (Lassance \& Sarriera, 2012), estas se diferenciam do Modelo de Carreira Caleidoscópica por não enfocarem na interrelação dos contextos e relacionamentos sobre a tomada de decisão profissional.

A literatura existente acerca dos pressupostos deste Modelo de Carreira demonstram que este pode ser utilizado para estimular o desenvolvimento de programas e políticas de Gestão de Pessoas, que proporcionem o aumento da produtividade e retenção de talentos, visto que, cada vez mais, colaboradores e colaboradoras estão buscando experiências de trabalho direcionadas para seus desejos e necessidades de Autenticidade, Balanço e Crescimento (Carraher, Crocitto, \& Sullivan, 2014; Hartung \& Subich, 2011; Sullivan \& Mainiero, 2007; 2008). Os estudos de Sullivan e Mainiero $(2007 ; 2008)$ e Cabrera (2009), demonstram que a Carreira Caleidoscópica pode ser utilizada como referência para o desenho de políticas e sistemas de gestão de pessoas, como oportunizar experiências internacionais para profissionais em início de carreira, atendendo suas demandas ligadas a Crescimento, por exemplo. Utrilla, Torraleja e Ortega (2012), salientam que, ao adotar políticas e sistemas de gestão ligados ao Modelo de Carreira Caleidoscópica, colaboradores atingirão altos níveis de satisfação com a carreira dentro da empresa, e tais benefícios individuais levarão à melhora de seu desempenho na organização.

O Modelo ainda permite a compreensão de carreiras fora das estruturas organizacionais tradicionais, como é o caso do empreendedorismo (Clarke, 2015; Tajlili, 2014; Sullivan et al., 2007), e em diferentes etapas do ciclo de vida (August 2011; Clarke, 2015; Carraher, Crocitto, \& Sullivan, 2014). Ademais, o modelo inova ao levar em conta a importância da conciliação entre papeis de vida para os indivíduos, conforme expresso no parâmetro Balanço (Braun et al., 2016; Lassance \& Sarriera, 2012), em contraste com outros modelos de carreira contemporâneos (Sullivan \& Baruch, 2015).

Apesar de sua relevância e potencial aplicabilidade, até 2009 as pesquisas envolvendo este construto foram somente teóricas ou qualitativas, utilizando os parâmetros como perspectiva holística, e não como variáveis a serem mensuradas. Essa questão foi solucionada com a investigação das diferenças geracionais em relação aos parâmetros do Modelo, para a qual foi desenvolvida uma escala com o objetivo de identificar as necessidades associadas aos parâmetros de Autenticidade, Balanço e Crescimento (Sullivan, Forret, Carraher, \& Mainiero, 2009). Neste estudo verificou-se que membros da Geração X - nascidos entre 1965 e 1983 - tinham demandas significativamente maiores de Autenticidade e Balanço em comparação aos Baby Boomers - nascidos entre 1946 e 1964. Em relação ao parâmetro Crescimento, não 
foram encontradas diferenças significativas entre as duas gerações. Por outro lado, pessoas com maior nível educacional e renda tiveram escores do parâmetro Crescimento mais elevado, enquanto mulheres com filhos vivendo em casa tiveram maiores níveis referentes a Balanço. Por fim, mulheres e profissionais com maior nível educacional também apresentaram escores superiores no parâmetro Autenticidade (Sullivan et al., 2009).

A criação da escala representou um avanço para estudos envolvendo o Modelo de Carreira Caleidoscópica, pois além de permitir a mensuração dos parâmetros que o compõem, representa uma possibilidade de instrumentalizar consultores e profissionais da área de planejamento de carreira (August, 2011; Grady \& McCarthy, 2008; Shaw $\&$ Leberman, 2015; Tajlili, 2014). No entanto, conforme apontado por Tajili (2014), a maioria dos estudos relativos ao Modelo de Carreira Caleidoscópica ainda derivam de suas criadoras, sendo fundamental gerar resultados de outros acadêmicos independentes, para verificar se as características previstas pelo modelo se apresentam da mesma forma em outros contextos e culturas.

Verifica-se, portanto, que o Modelo de Carreira Caleidoscópica tem sido utilizado como base para a compreensão e análise das decisões de carreira dos indivíduos. No entanto, não foram identificados estudos que utilizem de forma empírica o Modelo de Carreira Caleidoscópica no contexto brasileiro (Bandeira, 2017), fato que pode ser limitador para a disseminação do construto e para o uso de seus pressupostos no Brasil. Visto que brasileiros são mais passivos em relação à tomada de decisões de carreira em comparação à média global, conforme apontado em pesquisa da rede social Linkedin (2015), torna-se mais evidente a importância do uso de conceitos que permitam uma compreensão mais consciente e ativa de como são realizadas as escolhas profissionais, como é o caso do Modelo de Carreira Caleidoscópica.

Sendo assim, o presente estudo teve como objetivo geral adaptar e obter evidências de validade e fidedignidade da versão brasileira da escala dos parâmetros do Modelo de Carreira Caleidoscópica. O objetivo secundário foi explorar a diferenciação nos parâmetros em profissionais de diferentes perfis demográficos, segundo faixa etária, formação profissional, estar ou não rem relacionamento estável, ter ou não filhos e renda familiar.

\section{Método}

\section{Participantes}

O estudo de adaptação e o levantamento de evidências de validade da Escala de Parâmetros do Modelo de Carreira Caleidoscópica contou com uma amostra de 272 participantes, selecionados por conveniência, com idade entre 19 e 66 anos $(M=33,8 ; \mathrm{DP}=10,0)$, homens e mulheres, dos quais $56,6 \%$ eram do sexo feminino. Em relação à escolaridade, 19,1\% não possuíam formação de nível superior e 41,2\% tinham pós-graduação. Como critérios de inclusão na amostra, foram selecionados apenas indivíduos brasileiros maiores de idade que estivessem trabalhando no momento da coleta de dados e residindo no país de origem. O cálculo do tamanho amostral seguiu a recomendação de Hair, Tatham, Anderson e Black (2005) de que a razão entre o número de casos e a quantidade de variáveis seja igual ou maior do que cinco, assegurando resultados mais robustos.

\section{Procedimentos de adaptação do instrumento}

Para a adaptação e levantamento de evidências de validade da Escala de Parâmetros do Modelo de Carreira Caleidoscópica para o Brasil, inicialmente consultou-se a autora principal da escala original, tendo a mesma cedido autorização para estudo de adaptação do instrumento. No processo metodológico de adaptação foram seguidas as etapas sugeridas por Borsa, Damásio e Bandeira (2012), com $o$ intuito de prover, tanto evidências de equivalência semântica entre os itens da escala, quanto ao uso de propriedades psicométricas adequadas, conforme descrito a seguir:

a) Tradução do instrumento original: dois tradutores independentes, nativos na língua portuguesa e fluentes em inglês, realizaram a tradução da versão original da escala (Borsa et. al., 2012). Um dos tradutores era um expert em modelos de carreira, enquanto o segundo não estava familiarizado com os objetivos deste estudo. A escolha dos tradutores teve intuito de obter uma tradução com maior alinhamento científico no primeiro caso e uma tradução mais alinhada ao uso comum da língua portuguesa no segundo, por não ter sido influenciada pelo propósito acadêmico da pesquisa (Beaton, Bombardier, Guillemin, \& Ferraz, 2000).

b) Síntese preliminar do instrumento adaptada ao contexto brasileiro: essa síntese foi avaliada por dois juízes experts na área, buscando avaliar aspectos como a estrutura, instruções, escopo e adequação das expressões utilizadas na versão adaptada. Após essa etapa, a qualidade da versão em português foi avaliada também por oito representantes do público-alvo do instrumento, com o intuito de verificar se itens e instruções estavam claros, ou se deveriam ser reformulados para assegurar sua melhor adaptação ao contexto brasileiro.

c) Tradução reversa: Após a revisão da versão da escala em português, os itens foram traduzidos de volta para o idioma original do instrumento (inglês) por dois tradutores bilíngues, que não participaram da etapa anterior de tradução da escala original para o português. Essa etapa é chamada de back 
translation(Borsa et. al., 2012). As traduções em inglês foram sintetizadas em uma única versão e apresentadas para a autora do instrumento original, com o intuito de verificar e discutir se a nova versão era fidedigna aos objetivos da escala anterior.

d) Criação de novos itens: Após o consentimento da autora da escala original, iniciou-se um estudo piloto para obter evidências de validade do instrumento. Em uma análise preliminar com 90 participantes, verificou-se que os parâmetros Autenticidade e Balanço possuíam itens de baixa qualidade em relação à análise fatorial confirmatória de três fatores. Diante deste resultado, os pesquisadores optaram pela criação de três novos itens para o parâmetro Autenticidade e de um novo item para o parâmetro Balanço para a versão brasileira da escala, com base em revisão de literatura sobre o modelo. Após, procedeu-se a uma coleta de dados com novos participantes para a investigação das evidências de validadeda versão final do instrumento, composto pelos 15 itens originais acrescidos dos 4 novos itens criados para a versão brasileira do instrumento, perfazendo uma escala de 19 itens no total.

e) Procedimentos estatísticos: finalizada a etapa de sistematização do instrumento para o português, procedeu-se a um conjunto de operações estatísticas buscando o levantamento de indicadores de validade e aspectos de precisão da Escala de Parâmetros do Modelo de Carreira Caleidoscópica.

\section{Instrumentos}

a) Escala de Parâmetros do Modelo de Carreira Caleidoscópica. Versão adaptada do instrumento para o Português brasileiro, composta por quinze itens para o estudo piloto e por 19 itens no estudo final, para avaliar cada um dos três parâmetros (Autenticidade, Balanço e Crescimento). O instrumento utiliza uma escala Likert de cinco pontos, variando de "Isso não me descreve" (1) para "Isso me descreve muito bem" (5). Os índices de confiabilidade (Alfas de Cronbach) da escala original para Autenticidade, Balanço e Crescimento foram respectivamente $\alpha=, 76, \alpha=, 81$, e $\alpha=, 84$.

b) Questionário Sociodemográfico. Esse instrumento teve como objetivo avaliar os dados sociodemográficos dos respondentes. Por meio desse questionário foi possível levantar o perfil do participante - coletando informações como idade, formação profissional, relacionamento, filhos e renda familiar - para relacioná -los com as outras variáveis do estudo, demonstrando sua relação com os demais construtos analisados.

\section{Procedimentos éticos}

Todas as atividades foram submetidas ao Comitê de Ética da instituição a qual os pesquisadores estão vinculados e seguiram as Diretrizes e Normas de Pesquisa com Seres Humanos conforme Resolução no 466/2012 e Resolução n ${ }^{a}$ 510/2016 do Conselho Nacional de Saúde. É importante salientar que para participar da pesquisa, os respondentes precisavam realizar a leitura do termo de consentimento livre e esclarecido e expressar sua concordância em participar do estudo de livre e espontânea vontade. No mesmo endereço eletrônico no qual os participantes respondiam os instrumentos de pesquisa foi disponibilizada uma cópia do termo de consentimento informado que podia ser impressa, ao realizar o download de um arquivo em formato $P D F$.

\section{Procedimentos de coleta e análise dos dados}

A coleta de dados foi realizada por meio da aplicação de um questionário online, do aplicativo Qualtrics, no período de março a maio de 2017 . Foram enviados correios eletrônicos e mensagens nas redes sociais com o link para a pesquisa a indivíduos que atendessem aos critérios de inclusão da amostra, junto com uma breve descrição dos objetivos do estudo.O tempo médio de resposta ao instrumento foi de 8 minutos.

Após a coleta de dados, foi realizada a análise da estrutura fatorial da escala a partir de procedimentos fatoriais confirmatórios (Lloret-Segura, Ferreres-Traver, Hernández-Baeza, \& Tomás-Marco, 2014) e da consistência interna por meio do coeficiente ômega (Dunn, Baguley, \& Brunsden, 2014) das dimensões consequentes, buscando evidenciar sua validade e confiabilidade quando utilizada em estudos realizados no Brasil (Borsa et al., 2012). Os dados foram analisados com auxílio dos pacotes estatísticos SPSS (Statistical Package for the Social Sciences), JAMOVI (versão 0.8) e AMOS (Analysis of Moment Structures). Para levantamentos de indicadores concernentes ao construto, utilizou-se procedimento de análise fatorial confirmatória (Byrne, 2010), com método de estimação da máxima verossimilhança, com base na estrutura interna do instrumento. Utilizaram-se os indicadores de ajuste para os modelos confirmatórios: chi-quadrado ponderado (razão do chi-quadrado por graus de liberdade, $\chi 2 / \mathrm{gl}$ ) menor que 3 ; Comparative Fit Index (CFI), índice de Tucker-Lewis (TLI) e Root Mean Square Errorof Approximation (RMSEA). Para o cálculo da precisão das subescalas resultantes, recorreu-se à análise dos índices de confiabilidade (coeficiente alfa de Cronbach). 


\section{Resultados}

A Tabela 1 apresenta os itens originais (Sullivan et al., 2009) e sua versão adaptada ao português brasileiro. Também são apresentados os quatro itens criados pelos pesquisadores para versão brasileira do instrumento.

\section{Análise fatorial confirmatória}

Primeiramente, analisou-se o modelo original da escala (M1), composto pelos 15 itens correspondentesà versão original do instrumento, adaptados ao português brasileiro conforme apresentado na Tabela 1 , sendo consideradas as correlações entre as três dimensões latentes para análise. Como modelo alternativo (M2), testou-se uma versão concorrente do modelo original com acréscimo de quatro itens criados pelos pesquisadores.

O primeiro modelo (M1) não se adequou plenamente aos dados, apresentando os seguintes índices de ajustes: $\chi 2=219,216$, g.l. $=87(\mathrm{p}<0,001), \chi 2$ /g.l. $=2,520, \mathrm{CFI}$ $=0,848, \mathrm{TLI}=0,817, \mathrm{RMSEA}(90 \% \mathrm{CI})=0,075(0,063-$ $0,087)$. O modelo concorrente (M2) - com acréscimo de três itens na dimensão Autenticidade, um item na dimensão Balanço e mesma estrutura na dimensão Crescimento - também não foi plenamente ajustado. Os seus índices de adequação foram os seguintes: $\chi 2=329,973$, g.l. $=149$ $(\mathrm{p}<0,001), \chi^{2}$ /g. $1 .=2,21, \mathrm{CFI}=0,843$, TLI $=0,819$, RMSEA $(90 \% \mathrm{CI})=0,067(0,057-0,077)$.

Diante do não ajuste dos modelos M1 e M2, foram testados outros dois modelos com reespecificação dos erros das medidas, um considerando o modelo original de 15 itens (M3) e outro, o modelo adaptado de 19 itens (M4). As variáveis para covariância dos erros foram selecionadas a partir dos índices de modificação oferecidos pela saída de dados do AMOS, sendo escolhidas as variáveis (e4 - e5; e8 - e6 e; e14-e12). O modelo original reespecificado (M3) obteve os seguintes indicadores de ajuste: $\chi 2=200,042$, g.l. $=98(\mathrm{p}<0,001), \chi 2 / \mathrm{g} .1 .=2,04 \mathrm{CFI}=$ $0,889, \mathrm{TLI}=0,864$, RMSEA $(90 \% \mathrm{CI})=0,062(0,050-$ $0,074)$. Por sua vez, os índices de adequação do modelo adaptado reespecificado (M4) foram: $\chi 2=263,021$, g.l. $=$ $146(\mathrm{p}<0,001), \chi^{2} / \mathrm{gl}=1,80, \mathrm{CFI}=0,898, \mathrm{TLI}=0,881$, RMSEA $(90 \% \mathrm{CI})=0,054(0,044-0,065)$. A Figura 2 apresenta o diagrama do modelo adaptado reespecificado (M4), considerado aquele com os melhores indicadores de ajuste.

Finalmente, buscando avaliar a invariância do modelo segundo o sexo do respondente, testaram-se dois modelos adicionais com base na configuração estrutural de M4 (o modelo M5 com participantes do sexo masculino e; M6 com participantes do sexo feminino). Considerando a redução amostral para um tamanho inferior ao mínimo adequado para realização do procedimento confirmatório (Lloret-Segura et al., 2014), alguns indicadores de ajuste obtiveram valores abaixo dos níveis aceitáveis: para
M5 $-\chi 2=454,445$, g.l. $=292(\mathrm{p}<0,001), \chi 2 / \mathrm{gl}=1,54$, $\mathrm{CFI}=0,870, \mathrm{TLI}=0,847,(\operatorname{RMSEA}(90 \% \mathrm{CI})=0,045$ $(0,037-0,053)$; e para M6 $-\chi 2=452,445$, g.1 $=292(\mathrm{p}<$ $0,001), \chi^{2} / \mathrm{gl}=1,55, \mathrm{CFI}=0,870, \mathrm{TLI}=0,847$, RMSEA $(90 \% \mathrm{CI})=0,045(0,037-0,053)$. Sendo assim, observouse invariância estrutural segundo o sexo do participante.

Tomando o modelo adaptado reespecificado (M4) como mais adequado, calculou-se os indicadores de confiabilidade ômega $(\omega)$. Os valores obtidos foram: Autenticidade $(\omega=, 66)$, Balanço $(\omega=, 78)$ e Crescimento $(\omega=, 79)$.

\section{Estudo Comparativo}

Após a verificação de evidências de validade da Escala de Parâmetros da Carreira Caleidoscópica, foram feitas análises de diferenças de médias entre grupos. Este estudo comparativo foi realizado com os mesmos profissionais que participaram do estudo que buscou evidências de validade da Escala de Parâmetros da Carreira Caleidoscópica.

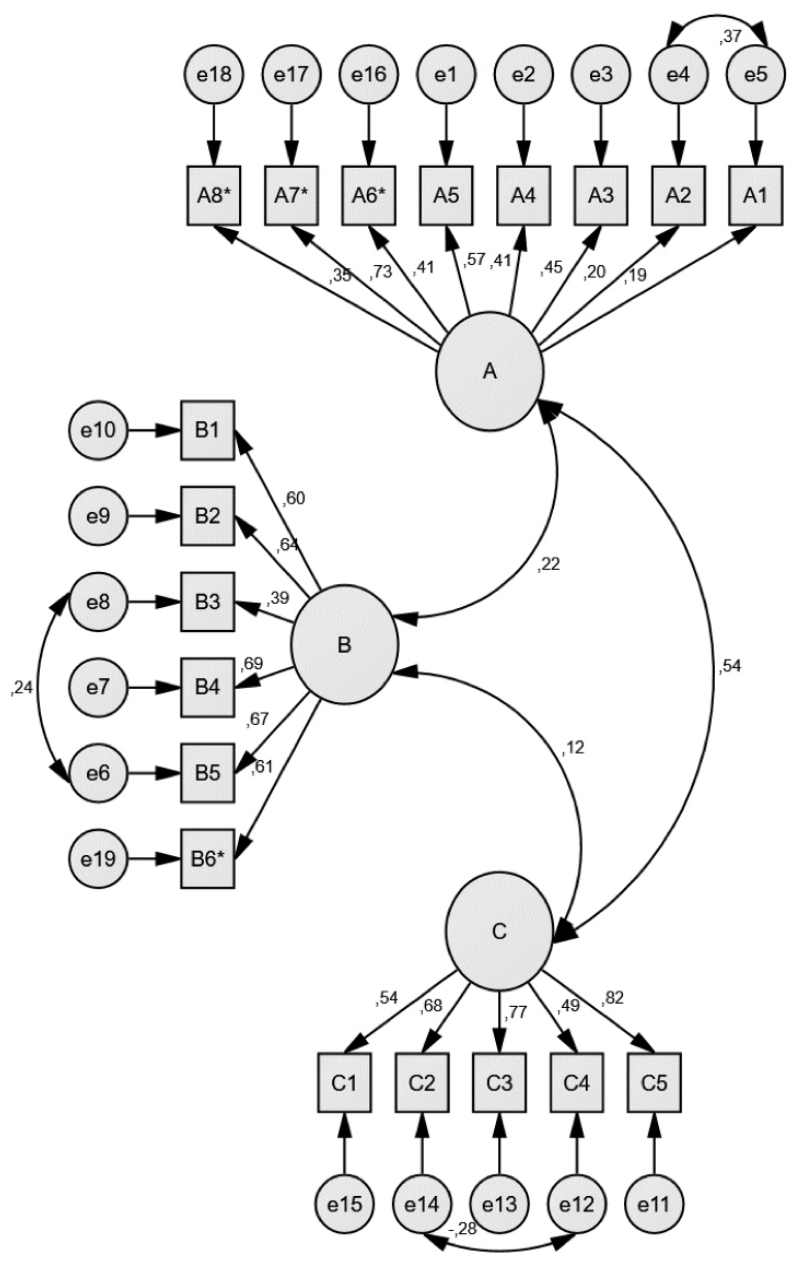

Figura 2. O Modelo de Carreira Caleidoscópica 
Tabela 1

Adaptação semântica dos itens

\begin{tabular}{lll}
\hline Versão original & Versão adaptada. \\
\hline & DIMENSÃO AUTENTICIDADE &
\end{tabular}

DIMENSÃO AUTENTICIDADE

A1 - I hope to find a greater purpose to my life that suits A1 - Eu espero encontrar um propósito maior para minha who I am. vida que possa combinar com quem sou.

A2 - I hunger for greater spiritual growth in my life.

A2 - Eu anseio por maior crescimento espiritual em minha vida.

A3 - I have discovered that crisis in life offer perspectives in ways that daily living does not.

A3 - Descobri que as crises que enfrentamos na vida oferecem perspectivas de uma forma diferente da vida cotidiana.

A4 - If I could follow my dream right now, I would.

A4 - Se eu pudesse seguir meu sonho neste exato momento, eu o seguiria.

A5 - I want to have an impact and leave my signature on A5 - Quero fazer a diferença e deixar minha própria marca what I accomplish in life naquilo que realizar em minha vida.

A6 - Se fosse necessário, eu abriria mão do sucesso ou da qualidade de vida para alcançar meus ideais.*

A7 - Quero fazer a diferença e trabalhar de acordo com o que acredito, mesmo diante das adversidades da vida.*

A8 - Prefiro não corresponder às expectativas dos outros do que abrir mão dos meus ideais.*

\section{DIMENSÃO BALANÇO}

B1 - If necessary, I would give up my work to settle prob- B1 - Se fosse necessário, eu abriria mão do meu trabalho lematic family issues or concerns. para resolver problemas ou preocupações relacionados a minha família.

B2 - Achieving balance between work and family is life's Holy Grail.

B2 - Alcançar o equilíbrio entre trabalho e família é algo que desejo muito, embora seja difícil fazer isso.

B3 - My work is meaningless if I cannot take the time to be with my Family.

B3 - Meu trabalho não faz sentido se eu não puder ter tempo para ficar com a minha família.

B4 - I constantly arrange my work around my family needs. B4 - Eu constantemente ajusto meu trabalho de acordo com as necessidades da minha família.

B5 - Nothing matters more to me right now than balancing work with my family responsibilities.

B5 - Nada é mais importante para mim neste momento do que conseguir equilibrar meu trabalho com minhas responsabilidades familiares.

B6 - Realizo decisões profissionais levando em consideração o impacto que terão em minha vida pessoal ou da minha família.*

\section{DIMENSÃO CRESCIMENTO}

C1 - Most people would describe me as being very goal- C1 - A maioria das pessoas me descreveria como alguém directed.

focado em seus objetivos.

C2 - I continually look for new challenges in everything I C2 - Eu constantemente procuro por novos desafios em do. tudo que faço.

C3 - I thrive on work challenges and turn work problems into opportunities for change.

C3 - Eu me desenvolvo diante de desafios profissionais e transformo problemas de trabalho em oportunidades de mudança.

C4 - I view setbacks not as "problems" to be overcome but C4 - Eu não encaro obstáculos como "problemas" a serem as "challenges" that require solutions. superados, mas sim como "desafios" que precisam de solução.

C5 - Added work responsibilities don't worry me.

C5 - Não me preocupo em ter mais responsabilidades no trabalho.

${ }^{\star}$ Itens criados para versão brasileira do instrumento. 
Dentro da amostra pesquisada, não houve diferenças significativas entre homens e mulheres em relação aos parâmetros da Carreira Caleidoscópica. No entanto, profissionais em relacionamentos estáveis obtiveram escores mais baixos para o parâmetro Autenticidade $[\mathrm{M}=3,43$, $\left.\mathrm{DP}=0,7 ; \mathrm{F}(193,1)=11,211, p=0,001, \eta^{2}=0,4\right]$ e mais elevados para Balanço $[\mathrm{M}=3,35, \mathrm{DP}=0,88 ; \mathrm{F}(193,1)=$ $\left.6,415, p=0,012, \eta^{2}=0,3\right]$, ainda que o fato de residir ou não com o/a companheiro/a não tenha apresentado diferença significativa em relação aos parâmetros. $\mathrm{O}$ mesmo ocorreu com profissionais que tinham filhos, que também apresentaram escores significativamente mais baixos para Autenticidade $[\mathrm{M}=3,32, \mathrm{DP}=0,67 ; \mathrm{F}(77,1)=9,235, p=$ $\left.0,005, \eta^{2}=0,4\right]$ e mais altos em relação a Balanço $[\mathrm{M}=$ $\left.3,52, \mathrm{DP}=0,94 ; \mathrm{F}(77,1)=8,136, p=0,003, \eta^{2}=0,4\right]$, indicando que o fato de ter filhos pode reduzir a importância do parâmetro Autenticidade e tornar mais evidente a orientação por Balanço nos indivíduos. Resultado similar foi encontrado na pesquisa realizada por Sullivan et al. (2009), em que mulheres com filhos registraram escores maiores para o parâmetro Balanço, ainda que no presente estudo, com amostra brasileira, não tenha sido identificada diferença segundo o sexo do progenitor.

A variável renda familiar apresentou correlação inversa com o parâmetro Autenticidade $(r=-0,185, p=$ $0,002)$, sugerindo que este parâmetro se torna mais proeminente quanto menor a renda familiar. Por outro lado, a formação profissional apresentou correlação positiva com o parâmetro Crescimento $(r=0,214, p<0,001)$, indicando que quanto maior a formação profissional do indivíduo, maior a orientação para este parâmetro, resultado também encontrado no estudo de Sullivan et al. (2009).

\section{Discussão}

O presente trabalho procurou adaptar e levantar evidências de validade e precisão para a Escala dos Parâmetros da Carreira Caleidoscópica no contexto brasileiro. Para atingir este objetivo, adaptou-se semanticamente o instrumento e testou-se a estrutura de três dimensões do construto com uma amostra de 272 profissionais, que indicou evidências de validade do instrumento. A versão final da escala apresentou 19 itens, distribuídos nos fatores previstos conforme resultados oriundos da análise fatorial confirmatória. Os indicadores de precisão do instrumento foram bons para os fatores Balanço $(\omega=, 78) \mathrm{e}$ Crescimento $(\omega=, 79)$, sendo o indicador de precisão do fator Autenticidade $(\omega=, 66)$ considerado regular (Dunn et al., 2014; Zanotti \& De Andrade, 2016). O estudo buscou ainda explorar diferenças nos níveis dos parâmetros da Carreira Caleidoscópica, conforme medidos pelo instrumento, entre profissionais de diferentes perfis demográficos.

Os resultados da busca por evidências de validade estão em consonância com a do instrumento original em inglês, apresentando na versão brasileira invariância estrutural segundo o sexo. Ademais, a análise dos escores dos parâmetros segundo os diferentes perfis demográficos também permitiu reforçar as evidências de validade do instrumento ao contexto brasileiro, visto que profissionais com vínculos familiares (com filhos ou em relacionamento estável) obtiveram escores significativamente maiores no parâmetro Balanço, enquanto profissionais com maior formação educacional apresentaram níveis mais elevados em relação ao parâmetro Crescimento. Tais achados vão ao encontro dos pressupostos teóricos do Modelo (Mainiero \& Sullivan, 2005; Shaw \& Leberman, 2015) e, portanto, corroboram para reforçar as evidências de validade do instrumento.

Reforça-se que os cuidados tomados na fase de tradução da escala, como o emprego de tradução reversa e análise por dois juízes independentes e pela autora do instrumento original em inglês, conferem uma razoável segurança quanto à compatibilidade semântica entre os itens originais e os da versão em português da escala. $\mathrm{O}$ uso de um estudo-piloto também permitiu que o instrumento fosse adaptado de forma mais adequada ao contexto brasileiro, especialmente no tocante ao parâmetro Autenticidade. Este fator foi o único que apresentou indicador de precisão considerado regular, e no estudopiloto foi o que apresentou menor qualidade em relação à análise fatorial confirmatória. Esse fato pode estar associado a aspectos culturais brasileiros em contraste com o que ocorre nos Estados Unidos, país de origem da escala original. Visto que o parâmetro Autenticidade está associado a ser fiel a si mesmo e a seus valores pessoais, definindo caminhos de carreira da forma mais autêntica e significativa possível, percebe-se que tal construto está fortemente ligado às expectativas individuais em relação à própria carreira.

Conforme apontam os indicadores do Índice de Hofstede (2017), o Brasil apresenta escore 38 na dimensão individualismo, ante um escore de 90 dos Estados Unidos. Essa dimensão representa o grau de interdependência que uma sociedade mantém entre seus membros, e se a autoimagem das pessoas é definida em termos de "Eu" ou "Nós" (Hofstede, 2017). Logo, tal resultado sugere que a orientação para valores individuais pode não ser tão significativa ou distinta no contexto brasileiro para a tomada de decisões de carreira com base no parâmetro Autenticidade - cuja definição corresponde à tomada de decisão baseada em valores pessoais (como explicitado em expressões dos itens da escala relacionados à "deixar minha própria marca", a "seguir meu sonho" ou "quero fazer a diferença"). É provável que aspectos associados à coletividade sejam mais associados à orientação para Autenticidade na cultura brasileira, em detrimento de valores individualistas. No entanto, a modificação da redação dos itens para a contemplação de valores sob um viés mais coletivo poderia comprometer o sentido original do 
parâmetro, desassociando-o de sua perspectiva individualista e consequentemente comprometendo as premissas da Carreira Caleidoscópica, bem como a possibilidade da realização de estudos transculturais utilizando a medida.

Tal inferência pode ser denotada também no estudo comparativo entre as médias dos grupos, visto que os escores para o parâmetro Autenticidade foram menores para indivíduos em relacionamentos estáveis e para aqueles que têm filhos. Neste sentido, reforça-se a percepção de que os itens referentes à Autenticidade estão associados a aspectos mais individualistas, e que têm sua importância reduzida ao passo que o papel familiar ou de cônjuge tornam-se mais salientes. Como esperado e apontado pela literatura, profissionais com filhos ou em relacionamentos estáveis apresentaram médias significativamente mais altas para os escores do parâmetro Balanço, indicando a importância da conciliação entre papeis de vida especialmente nas esferas familiar e laboral para estes trabalhadores (Braun et al., 2016; Lassance \& Sarriera, 2012).

Outro resultado interessante deste estudo refere-se à correlação negativa entre renda familiar e o parâmetro Autenticidade. Pode-se inferir que profissionais com menor rendimento sintam maior necessidade de buscar ocupações alinhadas a seus valores pessoais, visto que restrições decorrentes do baixo nível salarial possam representar obstáculos significativos para a satisfação desta demanda (Vale, Corrêa \& Reis, 2014). Já a correlação positiva entre o parâmetro Crescimento e formação profissional dos sujeitos denota que profissionais com maior qualificação tenham maior necessidade de evolução contínua e superação de desafios profissionais, possivelmente sendo a própria busca por maior qualificação profissional uma expressão deste parâmetro (Sullivan et al., 2009).

\section{Considerações finais}

Este estudo teve um objetivo essencialmente psicométrico: adaptar e verificar evidências de validade e fidedignidade para a versão brasileira da Escala de Parâmetros da Carreira Caleidoscópica. Pode-se concluir que os objetivos da presente pesquisa foram alcançados, uma vez que os indicadores de precisão do instrumento foram satisfatórios, e os resultados demonstram que a versão brasileira do instrumento, com 19 itens, pode ser utilizada em futuras pesquisas e mesmo em intervenções, ainda que de modo experimental, estando o instrumento em condições de ser empregado em novos estudos. Ademais, o uso da escala ainda contribui para a prática dos profissionais de aconselhamento de carreira, apoiando o exercício desta profissão no contexto brasileiro e ampliando a aplicação de seus pressupostos teóricos em um contexto culturalmente distinto do país de origem do instrumento (Hofstede, 2017; Tajlili, 2014). Ainda, a comparação entre grupos segundo as características sociodemográficas reforçou as evidências de validade do instrumento encontradas e permitiu uma análise mais aprofundada dos parâmetros que orientam as decisões de carreira, segundo os diferentes perfis de profissionais. No entanto, é necessário pontuar algumas limitações da pesquisa. Uma delas refere-se à escolha do método de amostragem por conveniência, sem haver o controle em relação à representatividade da população. Sendo assim, não é possível afirmar que indivíduos com características diferentes das dos profissionais que participaram deste estudo compreendam e respondam a escala da mesma forma. Também é importante salientar que os resultados obtidos podem estar relacionados às especificidades da amostra investigada, especialmente em relação ao contexto cultural dos locais de procedência dos participantes. Nesse sentido, sugere-se a realização de novos estudos com a Escala de Parâmetros da Carreira Caleidoscópica a fim de diversificar o perfil populacional das amostras e buscar novas evidências de validade do instrumento.

Ainda, faz-se necessário averiguar a relevância da Escala de Parâmetros da Carreira Caleidoscópica como instrumento para a área do aconselhamento de carreira. Em sua forma atual, a escala estimula o uso do construto como recurso heurístico nos processos de aconselhamento de carreira, sendo útil para a reflexão acerca dos parâmetros que orientam as decisões profissionais dos sujeitos. Ademais, por meio do uso dos pressupostos do Modelo e aplicação da escala com trabalhadores, é possível promover um ambiente de trabalho alinhado aos parâmetros de Autenticidade, Balanço e Crescimento. Conforme Sullivan e Mainiero (2007) pode-se promover a criação de programas de qualidade de vida ou o estímulo à participação dos funcionários em ações de responsabilidade social para trabalhar necessidades ligadas à Autenticidade; ofertar o serviço de creches no local de trabalho ou possibilitar o trabalho remoto para suprir demandas ligadas a Balanço; ou ainda organizar programas de mentoria ou políticas de job-rotation para atender questões associadas a Crescimento.

O incentivo a uma análise mais atenta e maior entendimento sobre os parâmetros que orientam as carreiras dos profissionais no Brasil pode contribuir ou incentivá-los na busca por seu desenvolvimento pessoal e laboral, fato que adquire especial importância diante de sua passividade em relação à busca por emprego (Linkedin, 2015; Cordeiro \& Albuquerque, 2016). O amplo espaço de aplicação e evolução da teoria demonstrado neste estudo ainda favorece a realização de novas pesquisas empíricas sobre as carreiras contemporâneas no contexto brasileiro (Sullivan $\&$ Baruch, 2009; Tajlili, 2014). Por fim, espera-se que os resultados alcançados permitam a exploração e melhor compreensão dos parâmetros que orientam as decisões de carreira dos brasileiros, contribuindo para a construção de trajetórias profissionais mais plenas e significativas. 


\section{Referências}

Ambiel, R. A. M. (2014). Adaptabilidade de carreira: uma abordagem histórica de conceitos, modelos e teorias. Revista Brasileira de Orientação Profissional, 15(1), 15-24.

Andrade, G. A., Kilimnik, Z., M., \& Pardini, D. J. (2011). Carreira Tradicional versus Carreira Autodirigida ou Proteana: um estudo comparativo sobre a satisfação com a carreira, a profissão e o trabalho. Revista de Ciências da Administração, 13(31), 58-80.

Audibert, A., \& Teixeira, M. A. P. (2015). Escala de adaptabilidade de carreira: evidências de validade em universitários brasileiros. Revista Brasileira de Orientação Profissional, 16(1), 83-93.

August, R. A. (2011). Women's later life career development: Looking through the lens of the kaleidoscope career model. Journal ofCareerDevelopment, 38(3), 208-236. doi:10.1177/0894845310362221

Bandeira, P. B. (2017). Motivações para a Carreira Empreendedora Segundo o Modelo de Carreira Caleidoscópica. Dissertação de Mestrado não publicada. Programa de Pós-graduação em Psicologia da Pontifícia Universidade Católica do Rio Grande do Sul. Porto Alegre, Brasil.

Baruch, Y., \& Bozionelos, N. (2010) Carrer Issues. In, Zedeck, S. APA Handbook of Industrial and Organizational Psychology, Volume 2: Selecting \& Developing Members for the Organization. Washington D.C.: American Psychological Association, 67-113.

Baruch, Y., \& Vardi, Y. (2015). A Fresh Look at the Dark Side of Contemporary Careers: Toward a Realistic Discourse. British Journal of Management, 27, 355-372. doi:10.1111/1467-8551.12107

Baruch, Y., Szucs, N., \& Gunz, H. (2015). Career studies in search of theory: the rise and rise of concepts. Career Development International, 20(1), 3-20. doi:10.1108/CDI-11-2013-0137

Beaton, D. E., Bombardier, C., Guillemin, F., \& Ferraz, M. B. (2000). Guidelines for the process of crosscultural adaptation of self-report measures. Spine, 25(24), 3186-3191.

Borsa, J. C., Damásio, B. R., \& Bandeira, D. R. (2012). Cross-Cultural Adaptation and Validation of Psychological Instruments: Some Considerations. Paidéia; 22(53), 423-432.

Braun, A. C., Vierheller, B., \& Oliveira, M. Z. (2016). Conflito trabalho-família em executivos: uma revisão sistemática de 2009 a 2014. Revista Brasileira de Orientação Profissional, 17(1), 19-30.

Byrne, B. M. (2010). Structural equation modeling with AMOS: basic concepts, applications, and programming (2a ed.). New York: Routledge, Taylor \& Francis.

Cabrera, E. F. (2007). Opting out and opting in: understanding the complexities of women's career transitions. Career Development International, 12(3), 218-237.

Cabrera, E. F. (2009). Protean organizations: reshaping work and careers to retain female talent. Career Development International, 14(2), 186-201.

Carraher, S. M., Crocitto, M. M., \& Sullivan S. (2014). A kaleidoscope career perspective on faculty sabbaticals. Career Development International, 19(3), 295 - 313.

Clarke, M. (2013). The organizational career: not dead but in need of redefinition. The International Journal of Human Resources Management, 24(4), 684-703.

Clarke, M. (2015). Dual careers: the new norm for Gen Y professionals? Career Development International, 20(6), 562-582.

Conselho Nacional de Saúde (2012). Resolução n. 466, de 12 de dezembro de 2012. Brasília.

Conselho Nacional de Saúde (2016). Resolução n. 510, de 07 de abril de 2016. Brasília.

Cordeiro, H. T. D., \& Albuquerque, L. G. (2016). Validação da escala de atitudes de carreira sem fronteiras e carreira proteana no Brasil. Revista de Carreiras e Pessoas, 6(2), 118-137.

De Andrade, A. L., Oliveira, M. Z., \& Hatfield, E. (2017). Conflito trabalho-família: um estudo com brasileiros e norte-americanos. Revista Psicologia Organizações e Trabalho, 17(2), 106-113. 
DeLuca, G., Rocha-de-Oliveira, S., \& Chiesa, C. D. (2016). Projeto e Metamorfose: Contribuições de Gilberto Velho para os Estudos sobre Carreira. RAC, 20(4), 458-476.

Duarte, M. E., Lassance, M. C., Savickas, M. L., Nota, L., Rossier, J., Dauwalder, J.-P., ... \& Vianen, A. E. M. (2010). A construção da vida: Um novo paradigma para entender a carreira no século XXI. Revista Interamericana de Psicología, 44(2), 392-406.

Dunn, T. J., Baguley, T., \& Brunsden, V. (2014). From alpha to omega: A practical solution to the pervasive problem of internal consistency estimation. British Journal of Psychology, 105(3), 399-412. doi:10.1111/bjop.12046

Hair, J. F., Tatham, R. L., Anderson, R. E., \& Black, W. (2005). Análise Multivariada de Dados. Porto Alegre: Bookman.

Hartung, Paul J., \& Subich, Linda M. (Eds). (2011). Developing self in work and career: Concepts, cases, and contexts. Washington: American Psychological Association.

Hofstede, G. (2017). Cultural Dimensions - Country Comparison. Disponível em: https://geert-hofstede.com/countries. html Acesso em: 07 de Julho de 2017.

Lassance, M. C. P., \& Sarriera, J. C. (2012). Saliência do papel de trabalhador, valores de trabalho e desenvolvimento de carreira. Revista Brasileira de Orientação Profissional, 13(1), 49-61.

Linkedin (2015). Brasil - Por que e como as pessoas mudam de emprego. Disponível em: https://business.linkedin.com/ $\mathrm{pt-br/talent-solutions/recursos/job-trends/por-que-e-como-as-pessoas-mudam-de-emprego-no-brasil \#} \mathrm{Acesso} \mathrm{em:}$ 02 de dezembro de 2017.

Lloret-Segura, S., Ferreres-Traver, A., Hernández-Baeza, A., \& Tomás-Marco, I. (2014). El análisis factorial exploratorio de los ítems: Una guía práctica, revisada y actualizada. Anales de Psicologia, 30(3), 1151-1169. doi:10.6018/ analesps.30.3.199361

Mainiero, L. A., \& Sullivan, S. E. (2005). Kaleidoscope careers: An alternate explanation for the "opt-out "revolution. The Academy of Management Executive, 19(1), 106-123.

Matos, D. C. M. D. (2015). Adesão ao Movimento OPT OUT: uma opção de transição de vida (Monografia). Universidade Federal do Rio Grande do Sul. Porto Alegre.

Oliveira, M. Z., Zanon, C., Silva da Silva, I., Pinhatti, M. M., Gomes, W. B., \& Gauer, G. (2010). Validação da Versão Brasileira da Escala de Atitudes de Carreira Sem-Fronteiras. Arquivos Brasileiros de Psicologia, 62(3), 1-114

Oltramari, A., Friderichs, B. P., \& Grzybovski, D. (2014) Carreira, família e a dialógica do assujeitamento: o discurso vigente em uma revista popular de negócios. Cadernos EBAPE.BR (FGV), 12, 112-130.

Peñaloza, V., Diógenes, C., G., \& Sousa, S. J. A. (2008). Escolha profissional no curso de administração: tendências empreendedoras e gênero. RAM. Revista de Administração Mackenzie, 9(8), 151-167.

Rosa, F. A. S., Zampier, M. A., \& Stefano, S. R. (2017). Tipos de carreira: análise da produção científica. Revista de Carreiras e Pessoas, 7, 358-373.

Shaw, S., \& Leberman, S. (2015). Using the kaleidoscope career model to analyze female CEOs'experiences in sport organizations. Gender in Management: An International Journal, 30(6), 500 - 515.

Sullivan, S. E., \& Baruch, Y. (2009). Advances in career theory and research: A critical review and agenda for future exploration. Journal of management, 35(6), 1542-1571.

Sullivan, S. E., \& Mainiero, L. A. (2007). Kaleidoscope careers: Benchmarking ideas for fostering family-friendly workplaces. Organizational Dynamics, 36(1), 45-62. doi:10.1016/j.orgdyn.2006.12.003

Sullivan, S. E., \& Mainiero, L. A. (2008). Using the kaleidoscope career model to understand the changing patterns of women's careers: Designing HRD programs that attract and retain women. Advances in Developing Human Resources, 10(1), 32-49. doi:10.1177/1523422307310110

Sullivan, S. E., Forret, M. L., Carraher, S. M., \& Mainiero, L. A. (2009). Using the kaleidoscope career model to examine generational differences in work attitudes. Career Development International, 14(3), 284-302.

Sullivan, S. E., Forret, M. L., Mainiero, L. A., \& Terjesen, S. (2007). What motivates entrepreneurs? An exploratory study of the kaleidoscope career model and entrepreneurship. Journal of Applied Management and Entrepreneurship, $12(4), 4$. 
Tajlili, M. H. (2014). A Framework for Promoting Women's Career Intentionality and Work-Life Integration. Career Development Quarterly, 62(3), 254-267.

Utrilla, P. N. C., Torraleja, F. A. G., \& Ortega, C. P. (2012). Nuevos retos en el desarrollo de carrera profesional: el modelo boundaryless career. Universia Business Review, 34, 14-35.

Vale, G. M. V., Corrêa, V. S., \& Reis, R. F. (2014). Motivações para o empreendedorismo: necessidade versus oportunidade?. Revista de Administração Contemporânea, 18(3), 311-327.

Wilkoszynski, C. C., \& Vieira, F. O. (2012). Carreiras Contemporâneas: Desafios e Contradições frente às Mudanças do Mundo do Trabalho. XXXVI Anais do EnAnpad. Rio de Janeiro: Anpad.

Zanotti, M. S., \& De Andrade, A. L. (2016). Avaliando pensamentos negativos sobre a carreira: O desenvolvimento de uma medida (EPNC). Revista Brasileira de Orientacao Profissional, 17(2), 175-187.

Recebimento: $14 / 07 / 17$

Aceite: $13 / 08 / 18$

Sobre os autores:

Patrícia Bock Bandeira é graduada em Administração pela Universidade Federal do Rio Grande do Sul e mestre em Psicologia Social no Programa de Pós-graduação em Psicologia da Pontifícia Universidade Católica do Rio Grande do Sul. Atua como pesquisadora associada e membro do Grupo de Estudos sobre Desenvolvimento de Carreira. Coordena atualmente o centro de ensino Cape Carreiras.

Alexsandro Luiz De Andrade é professor da Universidade Federal do Espírito Santo no nível de Graduação e Pós-graduação. Coordena atualmente o Laboratório de Avaliação e Mensuração Psicológica (Labamp).

Marcelo de Campos Velho Nora é graduado em Psicologia pela Pontifícia Universidade Católica do Rio Grande do Sul. Atualmente é membro do Grupo de Estudos sobre Desenvolvimento de Carreira, além de atuar como Orientador Profissional.

Marianna Marques Braga é graduada em Psicologia pela Pontifícia Universidade Católica do Rio Grande do Sul. Atualmente é membro do Grupo de Estudos sobre Desenvolvimento de Carreira, além de atuar como Consultora de Recursos Humanos na Consultoria A \& D Desenvolvimento de Pessoas e Organizações.

Manoela Ziebell de Oliveira é doutora em Psicologia pela Universidade Federal do Rio Grande Sul. Atualmente coordena o Grupo de Estudos sobre Desenvolvimento de Carreira no Programa de Pós-graduação em Psicologia da Pontifícia Universidade Católica do Rio Grande do Sul, além de atuar como consultora de carreira e membro do Núcleo de Pesquisa da Produtive Carreira e Conexões com o Mercado. 